\title{
CONGENITAL PERICARDIAL DEFECTS
}

\author{
BY \\ SYDNEY SUNDERLAND AND R. J. WRIGHT-SMITH
}

\begin{abstract}
From the Department of Anatomy and Histology, University of Melbourne, and the Department of Pathology, Royal Melbourne Hospital, Australia
\end{abstract}

Received October 10, 1944

Congenital pericardial defects are among the most uncommon of reported cardiac anomalies. In 1925 Moore was able to find 64 references to pericardial defects, but a detailed re-examination of the records by Southworth and Stevenson in 1938 reduced the number of confirmed cases in man to 52 and 7 of these occurred in monsters. They added another case to this series, and in a footnote to their paper referred to the case described by Dahl (1937) in which, however, a traumatic or pathological basis for the defect could not be definitely excluded: their review did not include Ladd's (1936) case. The purpose of this paper is to record the findings in two additional specimens. One is of especial interest in that the defect was directly responsible for the death of the patient. Such a result has been recorded on one previous occasion only.

It is not proposed to review the literature in any detail since this has already been comprehensively covered in excellent papers by Moore (1925), de Garis (1934), and Southworth and Stevenson (1938).

\section{REPORTS OF Two CASES}

Case 1. J. E. S., a boy, aged 2 years 2 months, was apparently healthy. He had been playing with an older boy when he complained of distress and ran to his mother holding the back of his head. He was rested, but still showed distress, and five hours after the onset refused his evening meal. A few hours later he became very restless and vomited. Fourteen hours after his first complaint, he suddenly collapsed and died. He did not receive any medical attention. An autopsy was performed eight hours after death. The body was that of a well-nourished male infant, well developed for his age. There were no external marks.

A large part of the parietal layer of the pericardial sac was absent (Fig. 1). The parietal layer present extended to enclose completely the right atrium and the greater part of the right ventricle. Anteriorly it showed a free, well-defined, constricted and rather rigid edge which crossed the sterno-costal surface of the heart from the root of the pulmonary trunk above to the inferior margin below, at a point well to the right of the apex. This free edge was situated to the right of the anterior interventricular sulcus and the interventricular branch of the left coronary artery. Inferiorly it was attached to the central tendon of the diaphragm between the inferior vena cava on the right and the line of the inferior interventricular sulcus, with its contained branch of the right coronary artery, on the left. Posteriorly it crossed the basal surface of the heart to the left of the pulmonary veins to reach again the left face of the root of the pulmonary trunk. The left ventricle and left auricle projected through this rigid orifice, the constricted edge of which had deeply grooved the surface of the heart. The mediastinal pleura on the left side below the hilum of the left lung also showed a large deficiency so that the left ventricle lay free in the left pleural cavity touching the median surface of the lower lobe of the left lung.

The surface and muscle of the right atrium were pale. The surface of the right ventricle was pale; the muscle was also pale, except at its apex, where it showed congestion and 


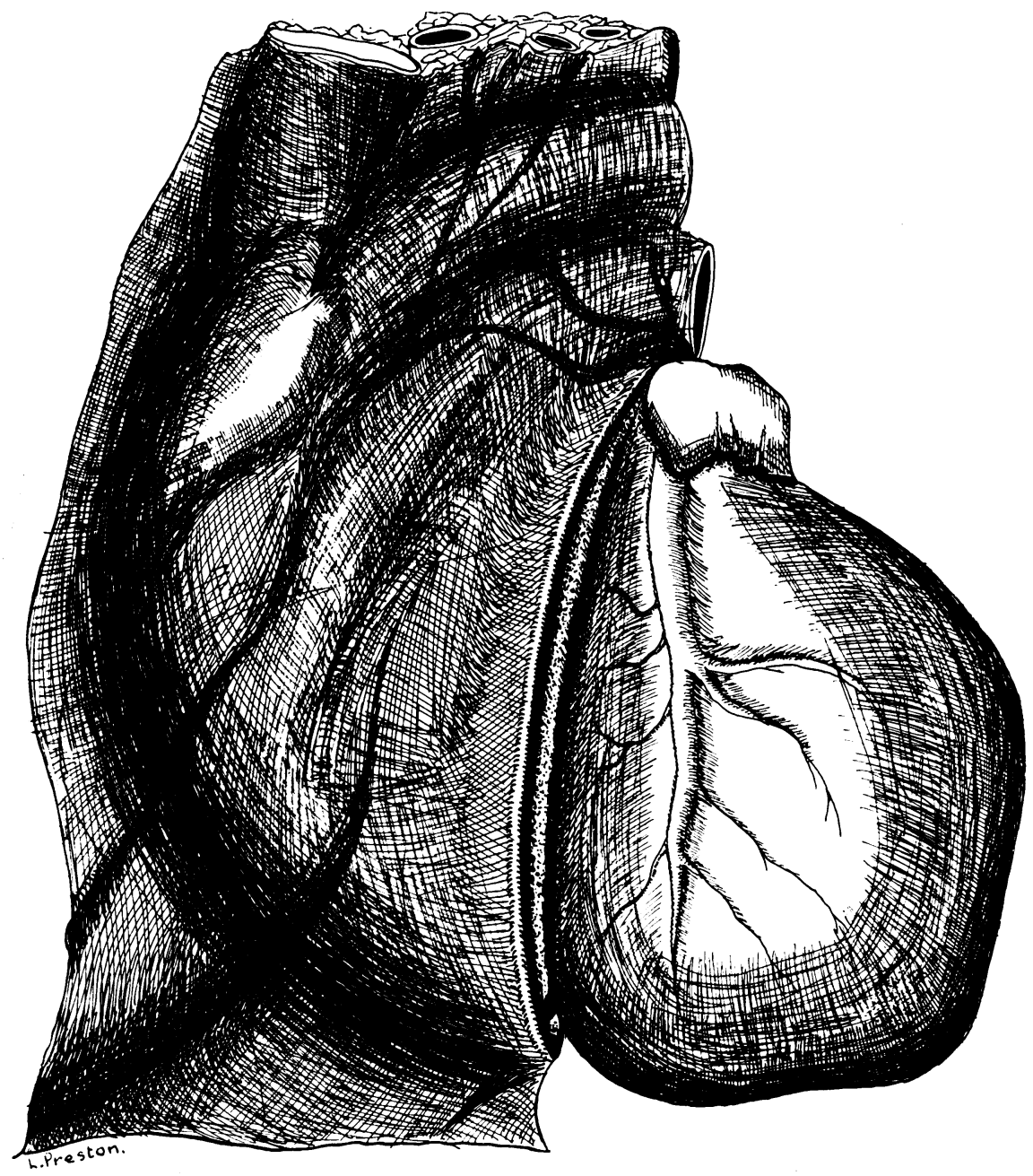

FIG. 1.-The heart and pericardium in Case 1 (see text).

hæmorrhage. The surface of the left ventricle was dark red and hæmorrhagic with many small focal hæmorrhages. The muscle of the left ventricle showed some hypertrophy. It was dark red in colour and at the base and apex there were large areas of hæmorrhage in the muscle tissue. The surface and muscle of the left atrium were very congested. The coronary sinus and great cardiac vein were distended with dark fluid blood. The cavities on the right were normal in size and contained red clot. On the left side, both the atrium and ventricle were dilated and contained red clot and fluid blood. The endocardium of the left ventricle showed some recent small hæmorrhages. The valves were normal. The foramen ovale was closed and there was no deficiency in the interventricular septum. The ductus arteriosus was patent though the lumen was constricted in the centre to about $2 \mathrm{~mm}$. in diameter. The aorta and its branches were normal.

The right pleural sac and the diaphragm were normal. The lungs were large and pale-red and showed a fine œdema in all parts. The bronchi and trachea were pale and filled with fine white froth. The thymus was not enlarged and the substance was pale-red and firm. The larynx, pharynx, and thyroid were normal. The alimentary tract and peritoneum were normal and the viscera showed no congenital abnormality. The liver was moderately congested and firm. The spleen was pale-red and soft. The pancreas and suprarenals were normal. The kidneys were congested and firm. The ureters and bladder were normal. The testes had not fully descended being just below the subcutaneous inguinal ring; they showed a normal structure. The bones of the skull were normal. The membranes of the brain were congested, and the brain was rather pale and firm, but showed no abnormality. 
Case 2. L. C. D., a man, aged 61 years, had had a stroke some two-and-a-half hours before admission to hospital, and could give no clear history. Examination revealed a wellnourished man showing deviation of the head and eyes to the right with tremor and flexion of the right arm and paralysis of the left arm and leg. He was treated as a case of cerebral thrombosis.

The temperature was $97 \cdot 8^{\circ} \mathrm{F}$., the pulse rate 70 , and the respiratory rate 20 . The blood pressure was $170 / 100$. The apex beat of the heart was in the left fifth intercostal space six inches from the midline. There was no right cardiac dullness and the sounds were regular and clear. Examination of the lungs and abdomen showed no abnormality. After some days moist sounds developed at the base of both lungs, and this was accompanied by elevation of the temperature and pulse rate. There was no improvement in the paralysis and the patient became incontinent. Six days after admission there was a sudden, rapid rise in the pulse rate and examination of the heart showed a " to and fro" murmur at the pulmonary area which was thought to be a friction rub. The systolic blood pressure fell to 150 ; the diastolic pressure remained at 100 . The temperature at this stage was $100 \cdot 4^{\circ} \mathrm{F}$., the pulse rate was 156 , and the respiratory rate 26 . The patient died eight days after admission. An autopsy was performed eight-and-a-half hours after death.

There was no pericardial sac in the true sense of the word (Fig. 2). The parietal layer was entirely deficient over most of the anterior surface of the heart, the whole of the apex, and much of the diaphragmatic and basal surfaces. It presented a free, loose margin, which was cord-like

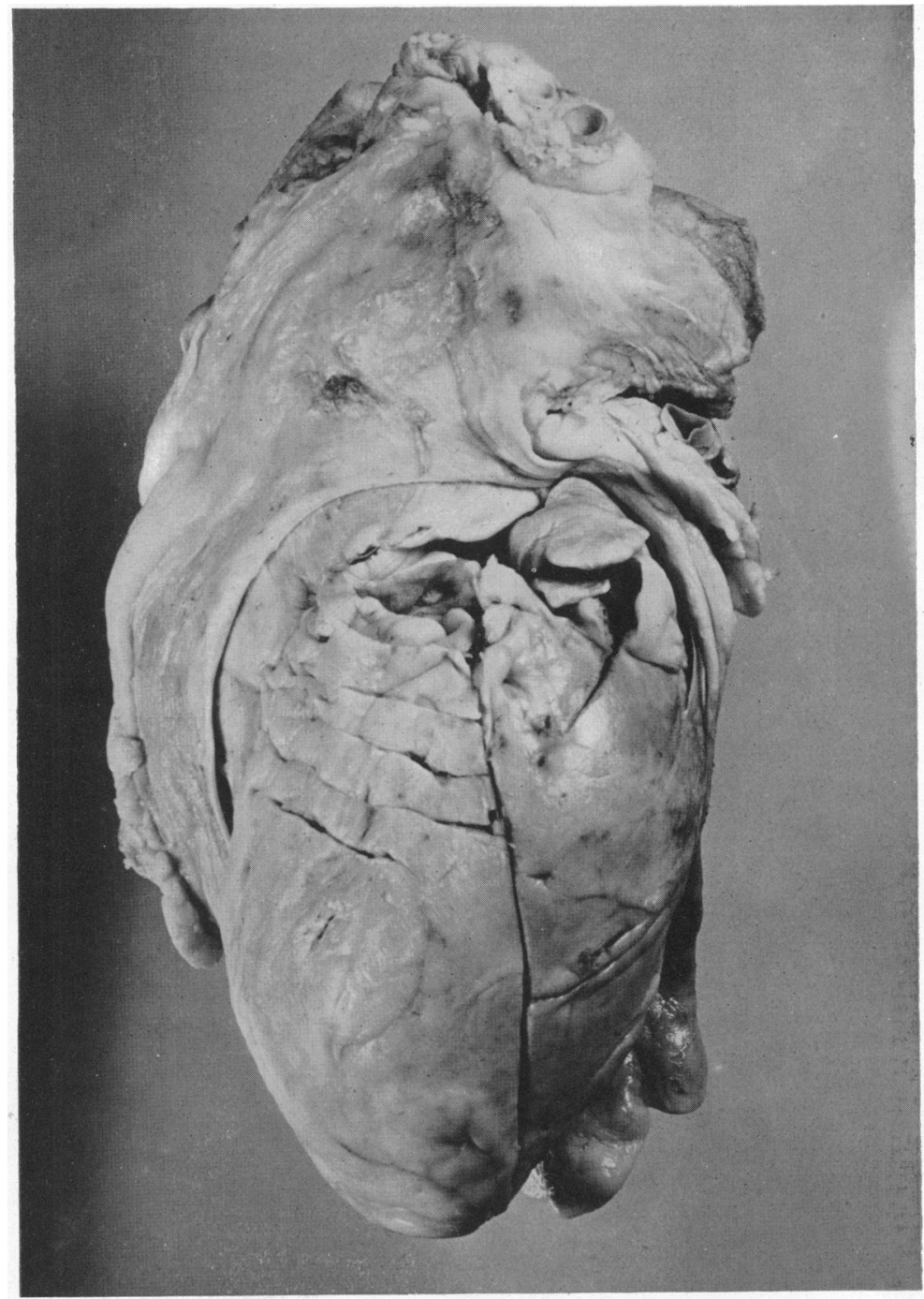

FIG. 2.-The heart and pericardium in Case 2 (see text). 
and smooth. Anteriorly the free margin appeared in the interval between the left auricle and the pulmonary trunk from which position it swung transversely to the right across the pulmonary trunk, the aorta, and the right coronary artery. It then curved downwards and crossed the sterno-costal surface of the right atrium, to the right of, and parallel to, the atrioventricular groove and the right coronary artery, to reach the right margin of the heart inferiorly. Turning round this margin it continued to the left, immediately anterior to the inferior vena cava, and was here attached to the central tendon of the diaphragm. It then ascended obliquely and to the left across the basal surface of the heart. On reaching the atrio-ventricular sulcus and the coronary sinus it accompanied these upwards to enter, from behind, the interval between the pulmonary trunk and the left auricle. Thus the greater portion of the heart was uncovered by parietal pericardium. Where present, this sheet was joined to the epicardium by scattered adhesions which prevented the free separation of the two structures. The parietal pleura was complete on both sides. The heart was large. It weighed $567 \mathrm{~g}$. (20 oz.). There was some hypertrophy of the left ventricle.

The coronary orifices were patent. The coronary arteries were thickened and atheromatous and showed patchy calcification. All branches were grossly narrowed. The left descending branch and the middle portion of the right coronary artery were completely occluded. The epicardium, apart from adhesions, appeared normal. The muscle of the left ventricle showed coarse fibrous scarring in the inner and middle layers. The endocardium of the left ventricle was white and densely fibrous. All cavities were dilated. The papillary muscles on the left side and the interventricular septum showed fibrosis. The valves were normal. The foramen ovale and the ductus arteriosus were closed. The aorta was large, thick-walled, and markedly atheromatous.

The lungs were large and the right lung presented four well-defined lobes. There was some early bronchopneumonic consolidation at both bases. The cerebral arteries were thickened and atheromatous and the basilar artery contained some pale thrombus. The brain showed large areas of softening and necrosis in the white matter of the right hemisphere. There were numerous small hæmorrhages in both hemispheres and in the pons. The other organs were normal.

\section{Development of the Pericardial Cavity}

The normal development of the pericardial and pleural cavities will be briefly outlined before proceeding to a discussion of the embryological basis of these defects.

During the first weeks of development, when the embryo is but $1.5-2.0 \mathrm{~mm}$. in length, irregular cavities appear in the mesodermal substance of the embryo, and these, by their enlargement and coalescence, form the primitive pericardial, peritoneal, and pleural cavities. The pericardial cavity is outlined by the fusion of a series of spaces that develop in the mesoderm surrounding the endothelial anlage of the developing heart to permit the effective functioning of that organ. In the lateral mass of body mesoderm the peritoneal cavity is similarly formed about the developing gut to permit intestinal movements.

A wedge of mesoderm, directed transversely across the embryo, is left interposed between these two cavities. This is the septum transversum and it contains the developing liver and the termination of the umbilical and vitelline veins. The septum does not, however, effect a complete separation between the two cavities, for these communicate dorsally, on either side of the mid-line, by the paired longitudinally-disposed pericardio-peritoneal canals. The developing lung buds ultimately herniate into these canals which fold about them, as development proceeds, to form the pleural sacs. With the formation of the head-fold the pericardial cavity comes to lie on the ventral side of the primitive pleural sacs.

The final stages of cœlomic differentiation are initiated at about the fifth week, when each pleural sac is sealed off from the pericardial cavity above and the peritoneal cavity below. Only the mechanism involved in the former process is relevant to this discussion. The separation is effected in the following manner. On each side the ductus Cuvieri establishes contact with and compresses the pericardio-peritoneal canal as it passes from the body wall 
to the heart. The pressure exerted by the vessel results in the formation of an elevation, the pulmonary ridge, which narrows the lumen of the canal. The most cranial portion of this ridge of tissue is intimately related to the opening between the pleural and pericardial cavities, and here it forms a valve-like membrane, the pleuro-pericardial membrane.

Some believe that pressure exerted by the growth of the ductus Cuvieri is alone responsible for closing the communication between the two cavities, while others claim that the communication is only partially constricted by the venous channel, and that its closure is finally completed by the active enlargement of the pleuro-pericardial membrane. It is difficult to assess the respective values of these two factors in effecting the closure, but it seems certain that both operate either in succession or coincidentally. It must not, however, be forgotten that, as growth proceeds and the pericardial and pleural cavities expand, the connection between the two, being an area of inactive growth, is gradually reduced to a minute foramen. It is upon this narrowed opening that the factors indicated above operate. The following, then, may be regarded as the essential factors responsible for the closure of the communication: (1) the relative reduction in the size of the opening consequent on the more active growth of neighbouring structures; (2) the active growth within the pleuro-pericardial membrane; and (3) the continued enlargement of the pleuro-pericardial membrane under the influence of the duct of Cuvier.

\section{INTERPRETATION OF THE DEFECTS}

Few attempts appear to have been made to account for the abnormality (Keith, 1907; Perna, 1909; Risel, 1911, 1912; Plaut, 1913; McGarry, 1914; Watt, 1931; and de Garis, 1934). It is generally accepted that the defect represents a persistence of the pleuro-pericardial foramen, but opinions are divided concerning the mechanism operating to prevent its normal closure. An examination of the records shows that the condition is almost exclusively leftsided. In one monster the defect was right-sided, and in another it was thought to be bilateral. Excluding the monsters, there was one human case in which the defect was possibly on the right and Moore (1925) has described a defect on this side in the dog. The defect varies in size from a small opening to one in which the entire left side of the pericardium is absent, thereby leaving the heart and lung occupying a common serous cavity. Any explanation advanced to account for the abnormality must satisfy these morphological features.

Keith (1907) claims, on the basis of observations on two specimens, that the pericardial defect " had been produced by the lung bud growing within and expanding the communication between the pericardium and pleura, for that communication lies immediately ventral to the point at which the lung bud appears. The condition should be described as dilatation of the pleuro-pericardial foramen." Keith, however, fails to explain why the lung bud herniates into the foramen and not into its normal position, or why the defects are almost exclusively left-sided. Though such an explanation as the one offered might conceivably account for the condition in his two specimens very few of the other recorded defects could be satisfactorily accounted for in this way. Perna (1909) and later Plaut (1913) advanced the theory that persistence of the communication is the result of the arrested development of the pleuropericardial membrane consequent on the premature obliteration of the left duct of Cuvier. On this basis, however, all defects should be left-sided. Moore (1925) also inclines to this view though the defect which he described was on the right side.

Risel (1912) disagreed with Perna's (1909) explanation on the grounds that there were extensive combined defects in the three monsters described by him (Risel) and that in one of them the pericardial defect was bilateral. He was content to attribute the defect solely to unspecified disturbances in general cœlomic differentiation occurring early in development. Such an explanation is too vague to be of value in explaining the majority of the defects recorded.

McGarry (1914) suggests that " the cells lining the cœlomic space, may at some period be 
particularly sensitive, and abnormal conditions occurring at this time, would result in disturbances, either an over-production or an under-production, of the serous derivatives " and attributes to this factor the lack of development of the pleuro-pericardial membrane. The abnormal conditions, however, are unspecified and he attempts to explain the almost exclusive incidence on the left as being due to the greater tension exerted on the pleuro-pericardial membrane by abnormal influences, induced by the asymmetry and rotation of the liver, which arrest its development. No evidence is advanced in support of this assumption.

Watt (1931) explains the defect as the result of the arrested development, from unspecified causes, of the pleuro-pericardial membrane-the subsequent enlargement of the communication being due to the presence of the heart or lung.

According to de Garis (1934) it is the heart that obstructs the closure of the foramen. He claims that the expansion of the heart tube to the left, which takes place during its enlargement and folding, is occasionally sufficiently great to prevent the pericardial, the pleural, or both these components of the pleuro-pericardial membrane, from growing forwards in their normal position. On this basis he explains all degrees of left-sided pericardial defect either with or without free communication between the pericardial and left pleural sacs. Though he cites as confirmatory evidence the fact that in many cases the heart is enlarged, elongated, or both, he maintains that " failure to find the adult heart abnormal in size or shape would not preclude the possibility that at a critical stage of development, when the pleuro-pericardial membrane grew ventrally and cranially, this growth was inhibited by the left-sided extension of the heart, which latter may or may not have been enlarged."

Thus all, with the exception of Risel, agree that the basis of the defect is obstruction of the normal development of the pleuro-pericardial membrane. Of the various theories advanced those of Perna (1909), Plaut (1913), and de Garis (1934) conform most closely to known embryological stages of development though they fail to account for those rare but confirmed cases in which the pericardium has been deficient on the right. Moreover, if the defect be attributed solely to the failure of the pleuro-pericardial membrane to develop, the pleural and pericardial cavities should remain in continuity in every case: but this is not always so. de Garis explains cases in which the pericardium alone is incomplete on the basis that the enlarging heart obstructs only the development of the pericardial aspect of the membrane.

There would appear, however, to be another factor operating in the production of these defects. The pericardium is obviously stretched as the pericardial cavity enlarges in order to accommodate the developing heart. Any opening in that enveloping sheet which is present when this process of stretching and enlargement sets in will be exaggerated unless factors normally leading to its closure operate at a much greater rate than that at which the opening is stretched. Consequently, if the foramen is to escape involvement when the pericardium is stretched it must close before the heart actively and rapidly enlarges. During the early stages of development it is conceivable that, in certain instances, the pleuro-pericardial foramen may be either unduly large, and/or its closure delayed or prevented, owing to (1) the arrested development or absence of the pleuro-pericardial membrane; (2) the failure of the membrane to grow at the rate required to close the foramen in time; (3) the absence or diminished effect of the differential growth factor which normally leads to its narrowing; or even (4) the direct influence of the enlarging heart as suggested by de Garis. Under these conditions the foramen is slowly widened as the pericardium is stretched by the enlarging heart and unless the factors responsible for its closure can, by their accelerated growth, make up lost ground it persists as a defect. Though the heart is entirely inside the pericardium in many of these abnormal cases, the defect present is obviously much larger than the original communication connecting the pericardial and pleural cavities. This lends support to the belief that the stretching to which the pericardium is subjected during development plays a significant role in maintaining and enlarging the foramen. Absence of the pleuro-pericardial membrane or obstruction to the closure of the pleuro-pericardial opening, acting singly or together, could hardly lead to an 
increase in size of the original communication. If, however, the heart is unduly large, which it appears to be in approximately half the cases, then direct pressure on the foramen by the enlarged heart could assist not only in keeping it open but also in actively dilating it.

The pericardial or both aspects of the pleuro-pericardial membrane may be involved in the manner indicated, thereby imparting to the defect those varieties in which the pericardium alone is defective or in which the pericardial and pleural cavities are in continuity. Moreover, during development both the right and the left pleuro-pericardial openings may be subjected to abnormal influences of the type described, which will result in pericardial defects. Naturally, however, such abnormalities will predominate on the left since there are additional factors operating on that side (obliteration of the duct of Cuvier, enlargement of the heart to the left, etc.), which may delay or prevent the closure of the foramen until the rapid growth of the heart can influence it.

The final size of the defect is determined by the original size of the foramen, the extent to which it is stretched by the enlarging heart, and the extent to which the factors attempting to close it are successful. The size of the defect at the time when the heart first begins to enlarge will also influence the degree to which that organ will project beyond the confines of the pericardium. The defect may never, at any stage, be sufficiently large to receive any portion of the heart that continues to develop entirely within the pericardium. This latter structure then shows, when development is completed, a defect of varying size, which is never sufficiently extensive to permit the heart to herniate through it when it expands physiologically or enlarges pathologically. On the other hand the foramen may be of such dimensions in the initial stages that the heart can protrude through it either partially or entirely. Under these conditions the organ may proceed to develop outside the pericardium in a common serous cavity with the left lung. The foramen is then progressively enlarged about the heart as it develops. Again, the defect may be of such a size when development ceases that, though the heart does not normally protrude through it, it may do so in the event of any enlargement produced pathologically or under conditions of extreme stress and exertion. If the heart be driven forcibly through the aperture in this way it may be constricted by the margins in such a manner as to affect adversely cardiac efficiency-in Boxall's case and the first case reported here the constriction was sufficiently severe to cause death.

Such a theory as that outlined accounts for both right- and left-sided defects, for the overwhelming predominance of the latter, and for all types and degrees of deficiency. The factors that appear to be responsible for the defect, acting either singly or in combination, may be conveniently summarized as follows.

(1) The normal stretching of the pericardium which takes place coincidentally with the enlargement of the heart.

(2) Premature and/or abnormal enlargement of the heart which precedes the closure of the pleuro-pericardial foramen. Projection of the heart through the opening at this stage effectively prevents any possibility of closure at a later date.

(3) An abnormally large foramen which is not fully closed when the heart and pericardium start to enlarge.

(4) Delayed or non-closure of a normal pleuro-pericardial foramen which is subsequently enlarged as the pericardium stretches to accommodate the developing heart. This arrested development may be due to: (a) defective formation of the pleuro-pericardial membrane as a result of premature obliteration of the duct of Cuvier or some other unidentified influence; (b) direct obstruction by the enlarging heart; or (c) failure or diminished activity of the differential growth factor by which the connection between the pleural and pericardial cavities is gradually reduced to a minute foramen.

(5) Failure of a membrane, whose development has been delayed, to grow sufficiently rapidly to close a defect which is being slowly extended as the pericardium expands to contain the enlarging heart. 


\section{Clinical Aspects of Pericardial Defects}

Apart from purely embryological considerations the clinical features associated with these defects are not without interest and may be briefly reviewed.

Side. Attention has already been directed to the almost exclusive incidence on the left. Both specimens reported in this paper were left-sided.

Type. Moore (1925) has classified the reported cases into the following three groups.

Group I. Those in which the heart and left lung occupy a common serous cavity. $\mathrm{He}$ records an incidence of 60 per cent in his series and Southworth and Stevenson (1938) one of 76 per cent in theirs.

Group II. Those in which there is only a foramen between the pericardial cavity and left pleural sac. He records an incidence of 21 per cent and Southworth and Stevenson (1938) one of 24 per cent. Both specimens reported in this paper would be included in this group though in the second case the defect involved the pericardium only.

Group III. Those in which there is either no trace of a pericardium or only rudiments thereof. He records an incidence of 19 per cent. Southworth and Stevenson (1938) believe that such a condition has never been conclusively demonstrated. This latter view is shared by the authors.

Associated Congenital Anomalies. Associated defects were multiple and extensive in the seven monsters. In six of the recorded series, associated anomalies in other organs were represented as follows: heart (2), lungs (1), pleural cavity (1), peritoneum (1), and kidney (1). However, as Southworth and Stevenson (1938) point out, the records of many cases are incomplete in that they contain references only to the cardiac condition. Associated defects were present in the two cases described in this report. In the first case the ductus arteriosus was patent and in the second the right lung was divided into four well-defined lobes.

Sex. From the reports that contain references to the sex it appears that the condition is approximately three times as common in males as in females. Both cases described in this report were males.

Influence on Expectation of Life. Southworth and Stevenson (1938), on the basis of a comparison of the mean age at death for the series of reported anomalies with the mean age at death for the United States area of registration for a given year, conclude that " the anomaly has no appreciable influence on life expectancy." In only two cases, that of Boxall and Case 1 in this paper, could death be attributed to the defect, and in neither was the condition diagnosed during life. Boxall's (1887) case was an adult in whom death occurred three days post-partum. Autopsy revealed a left-sided pericardial defect through which the heart had herniated and become strangulated. Death occurred suddenly with symptoms suggestive of a pulmonary embolism. It was suggested that changes in intrathoracic pressures following delivery were responsible for the fatal complication, but it is difficult to comprehend how this could have occurred three days after delivery when the heart was no longer subjected to the severe stress of labour.

Size of the Heart. From the limited number of cases containing a reference to the size of the heart, it would appear that, excluding those cases in which the enlargement was due to associated cardiovascular disease, the heart was enlarged in about 50 per cent of the cases.

Symptoms. Cardiac symptoms that could possibly be attributed to this abnormality were present in only three cases; there was a mild angina in two, while in the third adherent pericarditis was suspected during life and death was due to unexplained cardiac failure. The association between symptoms and defect in these three cases cannot, however, be regarded as conclusive. From an examination of the data contained in the case histories it may reasonably be inferred that pericardial defects are rarely the cause of cardiac symptoms. No symptoms directly attributable to the pericardial defects were present in the two cases here described.

Complications. It is interesting that in the two cases in which death could be attributed 
to the abnormality death was due to herniation of the heart through the defect, this being followed by strangulation. This, however, must be considered a rare event. On occasion, when the heart is partially outside the pericardium, pressure from the firm rim of the aperture may press on one or other of the coronary vessels and obstruct it. No such factor was operating in the second case described in this report. Though the heart is enlarged in 50 per cent of the cases there is nothing to suggest that this enlargement is due to the defect. Attention has frequently been directed, however, to the fact that continuity between the pleural and pericardial cavities exposes the heart to pulmonary infection (Abbott, White, Southworth and Stevenson, and others). There was a high incidence of pleuro-pericarditis in the recorded cases, and in 27 per cent death was due to pulmonary infection complicated by pleuro-pericarditis.

Diagnosis. Ladd (1936) observed the anomaly in a child aged two during the repair of a congenital diaphragmatic hernia. Dahl's (1937) patient developed a pneumopericardium during the establishment of a left pneumothorax which, according to him, could only be explained on the basis of a defect in the pericardium. Whether this was congenital or due to disease or trauma could not be ascertained. Excluding these cases the condition has never been recognized during life though Southworth and Stevenson (1938) carried out a detailed examination and investigation of the cardiac condition in a patient who was found to have a pericardial defect post-mortem. They conclude that "although the diagnosis is probably impossible in cases where the defect is represented only by a foramen, in those where there is a common cavity on the left the diagnosis may be suspected from (1) unexplained displacement of the heart to the left, and further confirmed if, in the absence of adhesions, there is (2) abnormal mobility of the heart. Unexplained cardiac enlargement (3), if present, would be further evidence in favour of the diagnosis, though its absence would not be significant." These conclusions are in general agreement with those reported by Maude Abbott (1927).

\section{SUMMARY}

Two cases in which post-mortem examination disclosed a congenital pericardial defect have been described. In neither case was the condition diagnosed or suspected during life. In one it was directly responsible for the death of the patient.

The normal development of the pericardial cavity has been briefly outlined.

The various theories advanced to account for the defect have been discussed and the limitations of each indicated. An alternative explanation has been advanced to account for all the morphological features displayed by the defect.

The clinical features of the condition have been briefly reviewed.

\section{REFERENCES}

An extensive bibliography has not been included. Such is provided in the papers of Grant, de Garis, Moore, and Southworth and Stevenson.

Abbott, M. (1927). Congenital Cardiac Disease, in Osler's Modern Medicine, Vol. 4. Philadelphia.

Boxall, R. (1887). Trans. Obst. Soc. Lond., 28, 209.

Dahl, E. (1937). Med. Rev. Bergen, 54, 312.

Grant, R. T. (1926). Heart, 13, 370.

Keith, A. (1907). J. Anat. Physiol., 41, 6.

de Garis, C. F. (1934). Anat. Rec., 59, 69.

Ladd, W. E. (1936). New Eng. J. Med., 214, 183.

Moore, R. L. (1925). Arch. Surg., 11, 765.

McGarry, R. A. (1914). Anat. Rec., 8, 43.

Perna, G. (1909). Anat. Anz., 35, 323.

Plaut, M. (1913). Z. Path., 12, 141.

Risel (1911). Dtsch. med. Wschr., 37, 2,405.

- (1912). Verhandl. dtsch. path. Gesellsch., 15, 379.

Southworth, H., and Stevenson, C. S. (1938). Arch. intern. Med., 16, 223.

Watt, J. C. (1931). Arch. Surg., 23, 996.

White, P. (1931). Heart Disease. New York. 